\title{
Targeted Cellular Delivery of Quantum Dots Loaded on and in Biotinylated Liposomes
}

\author{
Valeria Sigot, ${ }^{\dagger}$ Donna J. Arndt-Jovin, and Thomas M. Jovin* \\ Laboratory of Cellular Dynamics, Max Planck Institute for Biophysical Chemistry, 37077 Göttingen, Germany. Received \\ January 27, 2010; Revised Manuscript Received July 13, 2010
}

\begin{abstract}
We describe the preparation, biophysical characterization, and receptor-mediated cellular internalization of biotinylated lipid particles (BLPs) loaded on the surface and internally with two distinct (colors) of quantum dot (QD) probes. BLPs were formulated with 1.4 and $2.7 \mathrm{~mol} \% \mathrm{PEG}$-lipids containing either a fusogenic or $\mathrm{pH}-$ sensitive lipid to promote bilayer destabilization of endosomal membranes and favor QD cytoplasmic release. The amount of PEG was chosen to provide steric stabilization of the final construct. BLPs were loaded with a red-emitting $\mathrm{QD}_{655}$ and surface coated with a green-emitting $\mathrm{QD}_{525}$ conjugated to the epidermal growth factor (EGF) ligand in order to target the epidermal growth factor receptor (EGFR). The targeted and QD labeled BLPs showed strong and selective binding to EGFR-expressing tumor cell line and subsequent internalization. The dual-color QD labeling strategy and colocalization analysis allow prolonged live cell imaging of BLPs and loaded cargo independently, using a single excitation wavelength and simultaneous detection of both QDs. The chemistry of bioconjugation for the EGF ligand, the QDs, and the BLPs in a single lipid particle, involves only biotin-streptavidin interaction without requiring further purification from free EGF-QDs preformed complexes. Coupled with an encapsulated drug, the targeted and QD-labeled BLPs could provide imaging and drug delivery in a single multifunctional carrier.
\end{abstract}

\section{INTRODUCTION}

Liposomes that have been sterically stabilized by lipid derivatives of poly(ethylene glycol) (PEG) show persistence in the circulation, increasing the opportunities for specific, ligandand receptor-mediated targeting into tumor or disease-affected tissues in distal locations of the body $(1,2)$. Unwanted side effects of the PEG-coating include putative inhibition of liposome-cell membrane association and, intracellularly, avoidance of bilayer apposition and membrane fusion required for endosomal escape of the loaded cargo. PEG-lipid dissociation from the liposomes can be facilitated by incorporating fusogenic PEG-lipids with a short acyl chain promoting transfer from the liposome to the bilayer within minutes (3). Alternatively, PEG-lipid can be released intracellularly by adding a cleavable, $\mathrm{pH}$-sensitive PEG analogue in which the polymer moiety is cleaved from the liposome surface upon exposure to the acidic environment of certain endosomal compartments (4-8). An attractive type of unilamellar liposomes, referred to as stable plasmid lipid particles (SPLPs) by Wheeler and co-workers (9), are stabilized by $\mathrm{PEG}-$ ceramide lipids. They exhibit an average size of $70 \mathrm{~nm}$ and up to $70 \%$ encapsulation efficiency of plasmid DNA. The transfection competence of the SPLPs has proven superior after replacing PEG-Cer-C20 by the fusogenic ceramide with a shorter acyl chain PEG-Cer-C8 (10) or after adding a $\mathrm{pH}$-sensitive PEG-lipid in the SPLP formulation to trigger the intracellular plasmid release $(5,11)$.

Continuous cell imaging has been dramatically improved with the introduction of quantum dots (QDs), colloidal nanocrystals

* To whom correspondence should be addressed. E-mail: tjovin@ gwdg.de. Phone: 49(0)551-2011381/1382. FAX: 49(0)551-2011467. Laboratory of Cellular Dynamics, Am Fassberg 11, (37077) Göttingen, Germany.

$\dagger$ V. Sigot's present address is Centro Binacional Argentina-Italia de Investigaciones en Criobiología Clínica y Aplicada (CAIC). Universidad Nacional de Rosario. Arijón 28 bis (2000) Rosario, Argentina. with unique optical properties for long-term and multicolor imaging $(12,13)$. The broad absorption and narrow emission spectra of QDs allow the simultaneous detection of multiple colors of QDs upon illumination with a single light source (14). However, QD biocompatibility and potential toxicity remain critical issues for use in humans and thus currently limit their application to in vitro or animal models (15).

QDs have to be surface functionalized to act as useful biological probes. Targeting is usually achieved by conjugating a high-affinity ligand or antibody that provides preferential accumulation of the QD-ligand complexes, e.g., in a tumorbearing organ, in the tumor itself, in individual cancer cells, or in intracellular organelles $(13,16)$. The EGF receptor (EGFR) is an attractive target for drug and gene delivery, since it is overexpressed in a wide variety of human tumors, including brain, lung, liver, and breast cancer (17). It has been demonstrated that complexes of streptavidin-conjugated quantum dots (StAv-QDs) with biotinylated ligand EGF are biochemically competent ligands for EGFR (18). Protocols for binding biotinylated ligands to commercial streptavidin conjugated QDs have been described $(19,20)$. An important unresolved challenge is to effect the release of monodisperse QDs into the cytoplasm, considering that most of the delivery pathways involve endocytic routes leading to QDs trapped in endosomal compartments. A few reports have shown successful internalization of monodisperse QDs by osmotic lysis of pinosomes $(21,22)$ or microinjection (23), although in a nontargeted approach.

The development of tracking techniques combined with QD targeting or loading protocols allows for new strategies to monitor the uptake of drug delivery systems. Recent approaches for QD-labeled lipid-based delivery systems involve the embedding of hydrophobic QDs in lipid bilayers and micelles, or the encapsulation of hydrophilic QDs in liposomes, in order to significantly increase the cellular uptake of these fluorescent QD-lipid complexes ex vivo and in vivo (24-28). However, several studies have shown that nontargeted QDs micelles 
undergo unspecific uptake by cells and thus render them unsuitable for many applications (29).

Monitoring the behavior of liposomal-based drug delivery systems in living cells is a powerful approach for assessing the route and efficiency of drug delivery as well as the fate of the carrier after releasing the drug. At present, there are only a few published studies on lipid-based delivery systems combining QDs as imaging and targeting probes $(27,30,31)$. In our study, we explored the simultaneous application of two colors of QDs in a single lipid carrier, one QD as encapsulated cargo and the second as a receptor-targeting probe. We report here the preparation and characterization of biotinylated lipid particles (BLPs), loaded with $\mathrm{QD}_{655}$ and surface-modified with preformed complexes of EGF-QD ${ }_{525}$ mediated by biotin-streptavidin linkage. In this study, BLPs containing less than 3 mol \% PEG were formulated with either a fusogenic or a $\mathrm{pH}$-sensitive PEG-lipid in order to facilitate PEG transfer and to promote endosomal escape of $\mathrm{QD}_{655}$ into the cytoplasm. The EGF-QD ${ }_{525}$ BLP-QD 655 were targeted to A431 cells, a human epidermoid carcinoma line overexpressing the EGFR. Live cell imaging with fluorescence confocal microscopy demonstrated the specific binding and internalization of targeted BLPs by simultaneous detection of both QDs.

\section{EXPERIMENTAL PROCEDURES}

Chemicals. All reagents were of analytical grade. 1,2Dioleoyl-sn-glycero-3-phosphoethanolamine (DOPE), 1,2-dioleoyloxy-3-trimethylammoniumpropane chloride (DOTAP), 1,2-distearoyl-sn-glycero-3-phosphoethanolamine- $N$-[biotinyl(poly(ethylene glycol)) 2000] ammonium salt (biotin- $\mathrm{PEG}_{2000^{-}}$ DSPE), $N$-octanoyl-sphingosine-1-[succinyl (methoxy(poly(ethylene glycol) $) 750)]\left(\mathrm{PEG}_{750}-\mathrm{Cer}-\mathrm{C} 8\right)$, and 3-O-[2'-( $\omega$-monomethoxy(poly(ethylene glycol)) 2000) succinoyl]-1,2-distearoyl-snglycerol ( $\mathrm{PEG}_{2000}$-DSGS) were obtained from Avanti Polar Lipids (Alabaster, USA). Chloroform, ethanol, methanol, isopropanol, and water HPLC quality were purchased from Merck. 1-O-N-Octyl- $\beta$-D-glucopyranoside (OGP), HEPES, NaCl, sucrose, HABA (4-hydroxyazobenzene-2-carboxylic acid), biotin, and biocytin were obtained from Sigma and nonpolar polystyrene adsorbents Bio-Beads SM-2 from BioRad. Biotin-EGF (human recombinant) and streptavidin Alexa Fluor488 (ex. 488 $\mathrm{nm} / \mathrm{em} .519 \mathrm{~nm}$ ) were obtained from Invitrogen. Buffer solutions were of analytical grade and filtered through a $0.22-\mu \mathrm{m}$-sized membrane. Hepes Buffer Saline (HBS): 150 mM NaCl, $10 \mathrm{mM}$ Na-Hepes, pH 7.2.

Quantum Dots. Quantum dot streptavidin conjugates 525 $\mathrm{nm}\left(\mathrm{StAv}-\mathrm{QD}_{525}\right), 605 \mathrm{~nm}\left(\mathrm{StAv}-\mathrm{QD}_{605}\right)$, and ITK-carboxyl $\mathrm{QD}_{655}\left(\mathrm{QD}_{655}\right)$ with a CdSe core and $\mathrm{ZnS}$ shell were obtained from Invitrogen. QD with emission maxima at $655 \mathrm{~nm}$ have an ellipsoid core/shell with diameters of $6 \mathrm{~nm}$ (minor axis) by 12 $\mathrm{nm}$ (major axis). According to the manufacturer and determinations made in a parallel study (32), each StAvQD particle contains an average of 6-8 conjugated streptavidins.

Cell Lines and Reagents. A431 (human epidermoid carcinoma) and $\mathrm{CHO}$ (Chinese hamster ovary) cell lines were obtained from the American Tissue Culture Collection, and the melanoma cell line WM983A was kindly provided by Prof. Meenhard Herlyn (Wistar Institute, Philadelphia, PA). Dulbecco's Minimal Essential Medium, penicillin, streptomycin, and trypsin were obtained from Gibco. Leibovitz's L15 medium was from Mediatech, Inc. FBS was from PAN, and EDTA, BSA, and glucose were from Sigma. LabTek two-well glass chambers, plastic cell culture dishes, and flasks were purchased from Nunc or Sarstedt.

Preparation of Biotinylated Lipid Particles (BLPs). BLPs were prepared employing the detergent dialysis technique as described by Wheeler et al. (9) with modifications. For $1 \mathrm{~mL}$ of BLPs formulation, $8.4 \mu \mathrm{mol}$ DOPE (neutral lipid), $0.6 \mu \mathrm{mol}$ DOTAP (cationic lipid), and $0.024 \mu \mathrm{mol}$ biotin-DSPE were mixed in $2 \mathrm{~mL}$ glass vials. In individual vials containing this lipid mixture, $\mathrm{PEG}_{750}$-Cer-C8 and $\mathrm{PEG}_{2000}$-DSGS were added to obtain 1.4 and $2.7 \mathrm{~mol} \%$ PEG and a final concentration of $\sim 10 \mu \mathrm{mol}$ lipid/mL. These solutions were dried with a stream of argon and residual chloroform was removed under vacuum overnight.

The resulting lipid film was hydrated in $0.5 \mathrm{~mL}$ HBS and 0.2 M OGP detergent with continuous magnetic stirring at 60 ${ }^{\circ} \mathrm{C}$ to favor complete dissolution. After cooling the lipid-detergent mixture to RT, ITK-carboxyl $\mathrm{QD}_{655}$ were added in $0.5 \mathrm{~mL}$ HBS $\mathrm{pH} 7.4$ at $50 \mathrm{nM}$ final $\mathrm{QD}_{655}$ concentration. The mixture was immediately dialyzed against HBS using $10 \mathrm{kDa}$ MWCO dialysis cassettes (Slide-A-Lyzer from Pierce) for $1 \mathrm{~h}$ at RT and then dialysis continued in a cold room $\left(\sim 4{ }^{\circ} \mathrm{C}\right)$ overnight. After two buffer changes of $1 \mathrm{~L} \mathrm{HBS}$, residual detergent OGP was removed by adding polystyrene SM2 Biobeads (adsorbent capacity $\sim 117 \mathrm{mg} \mathrm{OGP/g}$ beads), and samples were loaded on top of a sucrose density gradient.

Ultracentrifugation in Sucrose Density Gradient. The sucrose gradient was prepared in $2.5 \mathrm{~mL}$ ultraclear thin-walled centrifuge tubes (Beckman) by carefully applying with a tip or syringe $0.6 \mathrm{~mL}$ layers of $2.5 \%, 10 \%$, and $20 \%$ (w/v) sucrose in HBS (10). The samples recovered from the detergent dialysis were adjusted to $1 \%(\mathrm{w} / \mathrm{v})$ sucrose and loaded on top of the gradient. Ultracentrifugation was carried out at $160000 \times g$ for $5 \mathrm{~h}$ at $10{ }^{\circ} \mathrm{C}$ in a TLS-55 swinging bucket rotor and a TL100 ultracentrifuge (Beckman). The resolved fluorescent bands observed under UV illumination were carefully recovered by manual pipetting and analyzed for QDs encapsulation, particle size, and biotin content.

TEM Analysis. For negative staining of BLPs, a solution containing 1:10 diluted samples was applied to a glowdischarged carbon-coated grid and stained with $1 \%$ uranyl acetate. Sample analysis was performed in a Philips $120 \mathrm{kV}$ BioTwin microscope equipped with a $1024 \times 1024$ pixels GATAN CCD camera.

Particle Size Analysis by Dynamic Light Scattering (DLS). The size of the BLPs was assessed by dynamic light scattering with a NanoZetaSizer particle analyzer (Malvern Instruments, UK) detecting the back-scattered light from a 633 $\mathrm{nm}$ laser at a fixed angle of $173^{\circ}$.

Samples were diluted in PBS, previously passed through 0.02 $\mu \mathrm{m}$ pore size filter to eliminate potential interfering impurities, and the measurements were performed at $25{ }^{\circ} \mathrm{C}$. The operating protocol was selected for spherical particles, applying 12 to 15 runs per measurement, which were automatically adjusted according to the concentration of particles in the sample, and three measurements were performed on each sample. The autocorrelation function for size distribution was calculated using the CONTIN mathematical approach for heterodisperse, polydisperse, and multimodal systems.

The mean hydrodynamic diameter obtained represents only an intensity-based average value and does not give any information on the prevailing size distribution. For this reason, the polydispersity index (pdi) is also stated in order to provide information about deviation from a monomodal distribution (pdi $\leq 0.25$ ).

Biotin Quantitation. The biotin content of BLPs was determined on samples recovered after detergent dialysis and ultracentrifugation in sucrose gradient employing a fluorometric assay (33). A standard curve was obtained by titrating $50 \mu \mathrm{L}$ of a solution containing $25 \mathrm{nM}$ StAv-Alexa488 and $125 \mu \mathrm{M}$ HABA in HBS with biocytin added from an aqueous stock solution to final concentration of $0-200 \mathrm{nM}$. The fluorescence of standards and samples was measured in a spectrofluorometer 
(Varian Cary Eclipse); Alexa Fluor488 dye was excited at 485 $\mathrm{nm}$ and the emission spectra were collected between 500 and $700 \mathrm{~nm}$. The fluorescence intensity at the emission maximum $(519 \mathrm{~nm})$ was plotted as a function of biocytin concentration, and the data were fitted to a sigmoidal curve to obtain a calibration curve, from which the corresponding values for the unknown samples could be derived. Under the conditions assayed, a $\sim 6$-fold increase in fluorescence signal upon complete displacement of HABA was observed with a detection limit of approximately $3 \mathrm{nM}$ biocytin (Supporting Information).

Cell Culture. A431 and CHO cell lines were cultured in Dulbecco's Minimal Essential Medium (D-MEM) supplemented with $10 \%$ fetal calf serum (FCS), $50 \mathrm{U} / \mathrm{mL}$ of penicillin (Pen), and $50 \mathrm{U} / \mathrm{mL}$ of streptomycin (Strep). WM983A cells were grown in three parts DMEM and one part Leibovitz's L15 medium supplemented with $10 \%$ heat inactivated $\left(56{ }^{\circ} \mathrm{C}\right.$ for $20 \mathrm{~min}$ ) FCS and Pen-Strep. All cells were grown as a monolayer at $37{ }^{\circ} \mathrm{C}$ in a humidified atmosphere containing $5 \%$ $\mathrm{CO}_{2}$. For microscopy experiments, cells were seeded one or two days in advance onto 12-mm-diameter glass coverslips or twowell glass chambers and employed at a 40-50\% confluence. For binding experiments, cells were starved in DMEM for $4 \mathrm{~h}$ prior to the incubation with EGFR targeted BLPs to reduce signaling induced by growth factors present in the serum.

Labeling and Targeting of BLPs with EGF-QDs Preformed Complexes. According to the manufacturer and confirmed in other studies, the StAv-QDs contain 6-8 streptavidins per QD. Biotin-EGF was coupled to StAv-QD ${ }_{525}$ at a ratio of $4: 1$ in HBS with $0.5 \%$ BSA at final concentration of $50 \mathrm{nM}$ QDs. Solutions were incubated at $15^{\circ} \mathrm{C}$ for at least $30 \mathrm{~min}$ with gentle agitation before coupling to BLPs.

BLPs with encapsulated $\mathrm{QD}_{655}$ were incubated with preformed complexes of EGF-QD 525 in HBS supplemented with $0.5 \%$ BSA. The StAv- $\mathrm{QD}_{525}$ concentration was $20 \mathrm{nM}$, whereas biotin concentrations of BLPs varied from $0.4-0.8 \mu \mathrm{M}$ depending on the formulation. This mixture was incubated for at least $2 \mathrm{~h}$ at $15{ }^{\circ} \mathrm{C}$ with continuous shaking and diluted to 1 or $2 \mathrm{nM} \mathrm{QD} \mathrm{QD}_{525}$ in Tyrode's buffer for live cell experiments by confocal fluorescence microscopy (see Table S1 in Supporting Information). Separation of EGF-QD-BLPs from EGF-QD complexes by size exclusion chromatography or sucrose gradient (data not shown) led to unacceptable sample loss.

Internalization Studies. A431 cells were seeded onto glass coverslips $\left(2 \times 10^{5}\right.$ cells $\left./ \mathrm{mL}\right)$. At $40-50 \%$ confluence, cells were serum starved for $4 \mathrm{~h}$ before adding the BLPs. The QD labeled and targeted BLPs (EGF-QD ${ }_{525}-\mathrm{BLPs}_{-} \mathrm{QD}_{655}$ ) were diluted in 200 or $500 \mu \mathrm{L}$ Tyrode's buffer supplemented with $0.5 \% \mathrm{BSA}$ and $20 \mathrm{mM}$ glucose and added to cells to obtain final concentrations of $0.5 \mathrm{nM}$ QD525 and $2 \mathrm{nM}$ EGF. Starved cells were precincubated with targeted and nontargeted BLPs at $10{ }^{\circ} \mathrm{C}$, a temperature nonpermissive for endocytosis. Then, cells were warmed and incubated 10 and $60 \mathrm{~min}$ at $37{ }^{\circ} \mathrm{C}$. Finally, unbound BLPs were removed and cells rinsed with Tyrode's buffer. Live cell imaging by confocal microscopy was carried out immediately after removing the complexes, for up to $2 \mathrm{~h}$ at RT $\left(\sim 20^{\circ} \mathrm{C}\right)$. In another set of experiments, cells were incubated with the BLPs during a 10 min pulse at $37{ }^{\circ} \mathrm{C}$ and QDs were chased after 4 and $12 \mathrm{~h}$ incubation at $37{ }^{\circ} \mathrm{C}$. When incubation at $37{ }^{\circ} \mathrm{C}$ exceeded $1 \mathrm{~h}$ after removing the BLPs, Tyrode's buffer was replaced by complete D-MEM.

Competitive Binding Assay. For competitive binding experiments, free EGF (recombinant human) was added to starved A431 cells at a final concentration of $200 \mathrm{ng} / \mathrm{mL}(33 \mathrm{nM})$ and cells were incubated for $5 \mathrm{~min}$ at $10{ }^{\circ} \mathrm{C}$. EGF-QD $605-\mathrm{BLPs}-$ $\mathrm{QD}_{655}$ were added to a final concentration of $0.5 \mathrm{nM} \mathrm{QD}_{605}$ and $2 \mathrm{nM}$ EGF and incubation resumed for $10 \mathrm{~min}$ at $37^{\circ} \mathrm{C}$. Cells were washed with Tyrode's buffer, and confocal microscopy was initiated immediately and performed for $60 \mathrm{~min}$ at RT.

Incubation of BLPs with EGFR Negative Cells. EGF$\mathrm{QD}_{605}$-BLPs-QD 655 were added to $\mathrm{CHO}$ and WM983A cell lines, both devoid of EGF receptor, at a final EGF concentration of 2 $\mathrm{nM}$ and incubation proceeded for $5 \mathrm{~min}$ at $10{ }^{\circ} \mathrm{C}$, followed by $10 \mathrm{~min}$ incubation at $37^{\circ} \mathrm{C}$. Cells were then washed with Tyrode's buffer and images were acquired immediately during $60 \mathrm{~min}$ at RT by confocal microscopy.

Simultaneous Tracking of Two Different Colors of QDs. Confocal fluorescence microscopy was performed with a Zeiss 510 confocal laser scanning microscope using a water immersion objective C-Apochromat $63 \times 1.2 \mathrm{NA}$. The simultaneous excitation of $\mathrm{QD}_{525}$ and $\mathrm{QD}_{655}$ was achieved with the $488 \mathrm{~nm}$ laser line, or in some experiments with the $458 \mathrm{~nm}$ line to increase the fluorescence intensity. QDs signals were detected simultaneously and collected in separated channels with appropriate filters, in this case, 520/20 BP for QD $_{525}$ and 585 LP for $\mathrm{QD}_{655}$. When $\mathrm{QD}_{525}$ was replaced by $\mathrm{QD}_{605}$ in preformed complexes with biotin-EGF, the $\mathrm{QD}_{605}$ and $\mathrm{QD}_{655}$ were detected using 585-615 BP and 650 LP filters, respectively. To eliminate possible crosstalk when using the same excitation wavelength, single color EGF-QD controls were tested for optimization of the gain settings. Confocal planes were acquired with a pinhole set to 1 Airy disk. With the given settings, channel crosstalk was negligible as determined using single-labeled samples.

$Z$-series were taken at $10.4 \mu \mathrm{m}$ intervals and deconvoluted using Huygens image processing software (Scientific Volume Imaging, Hilversum, The Netherlands) followed by 3-D reconstruction. For qualitative analysis, images were backgroundcorrected and contrast stretched; fluorescence intensity profile and 3-D reconstruction were performed with the software Image $J$ (NIH).

\section{RESULTS}

Encapsulation of QDs in Biotinylated Lipid Particles (BLPs). The detergent dialysis technique was explored for the encapsulation of ITK-carboxyl QD 655 . The PEG-lipid percentages selected for BLP formulation were the lowest that allowed the self-assembly of BLPs without forming aggregates during detergent dialysis. Since we were interested in the cytoplasmic delivery of $\mathrm{QD}_{655}$, the PEG shield should be disrupted in the endosomes. To promote PEG-lipid transfer out of the BLP bilayer and membrane fusion required for endosomal escape, the fusogenic PEG-Ceramide-C8 and $\mathrm{pH}$-sensitive lipid PEGDSGS were included. Three BLPs formulations were prepared with $1.4 \%$ and $2.7 \%$ PEG $_{750}$-Cer-C8 and $1.4 \%$ PEG $_{2000}$-DSGS in combination with $84 \%$ DOPE, $6 \%$ of the cationic lipid DOTAP, and $0.24 \%$ of biotin-DSPE. The $\mathrm{QD}_{655}$ were added to the hydrated mixture of lipids and OGP detergent in $10 \mathrm{mM}$ HBS pH 7.4, and the solutions were dialyzed against HBS.

After detergent dialysis a lipid recovery of $58 \pm 14 \%$ was estimated from the amount of incorporated biotin-DSPE measured by a fluorometric assay (see Supporting Information) in three independent BLPs preparations. To separate and recover QDs loaded BLPs from less dense empty BLP and nonencapsulated QDs, the samples obtained after the detergent dialysis were loaded on top of a discontinuous density gradient of $2.5 \%, 10 \%$, and $20 \%$ sucrose and ultracentrifuged. Three well-defined fluorescent bands were observed (Figure 1A, top right): a bright fluorescent band on top (fraction I), a broader and less intense fluorescent band underneath (fraction II), and a fluorescent pellet (fraction III). Biotin incorporation was detected in fractions I and II but not in the fluorescent pellet, indicating the separation of free and more dense QDs from lipidcontaining particles. The presence of QDs in the less dense and brighter band (fraction I) may have resulted from electrostatic 


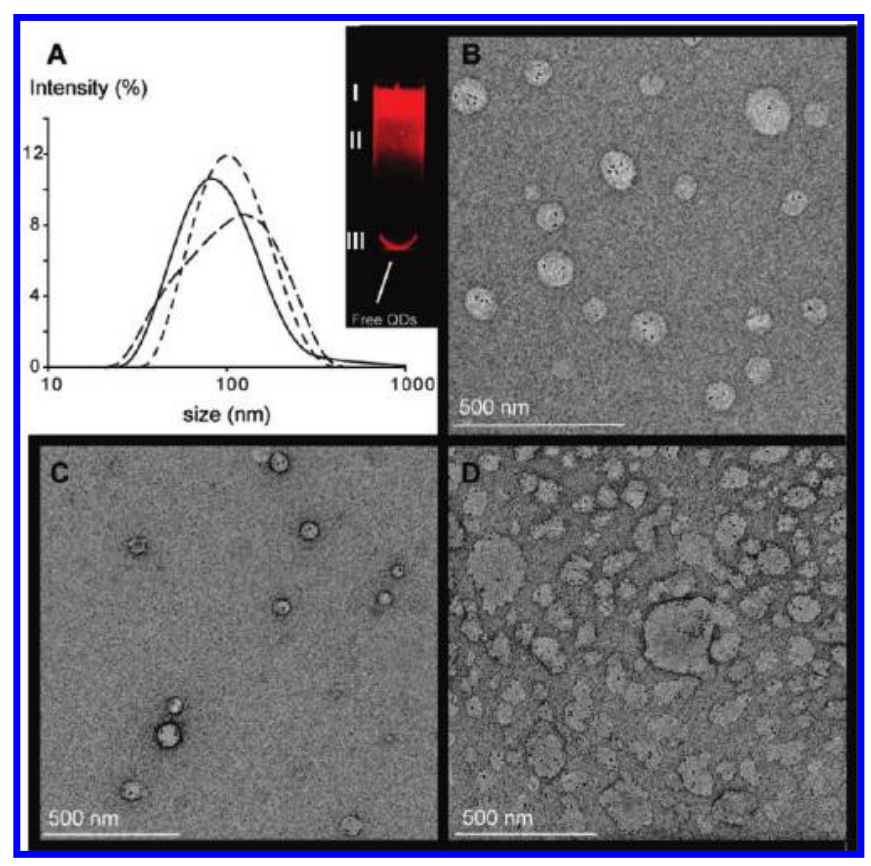

Figure 1. Size characterization of $B L P-Q_{655}$ after purification in sucrose density gradient. (A) (Top right) $B L P-Q_{655}$ recovered in fluorescent fractions II, whereas free QDs appeared mainly in the pellet (fraction III). (Top left) representative size distribution of BLP-QD 655 measured in fraction II by DLS (- - ) $1.4 \%$ PEG $_{750}-\mathrm{Cer}-\mathrm{C} 8,(-) 2.7 \%$ $\mathrm{PEG}_{750}$-Cer-C8, and (- -$) 1.4 \% \mathrm{PEG}_{2000}$-DSGS. TEM analysis of $\mathrm{QD}_{655}$ encapsulated in BLPs, (B) $1.4 \% \mathrm{PEG}_{750}$-Cer-C8, (C) $2.7 \%$ $\mathrm{PEG}_{750}$-Cer-C8, (D) $1.4 \% \mathrm{PEG}_{2000}$-DSGS. Scale bar $500 \mathrm{~nm}$.

interactions between the carboxylic groups on QD surfaces and the amino groups in free, nonincorporated DOTAP. Because this fraction may have contained empty vesicles (9), it was not characterized further.

Size Analysis of QD-Loaded BLPs. The presence of BLPs in fraction II was confirmed by size analysis for all lipid formulations. Figure 1A shows the size distribution of BLP$\mathrm{QD}_{655}$ as determined by DLS. The mean hydrodynamic diameter was $106 \mathrm{~nm}$ (pdi $=0.2$ ) for BLPs with $1.4 \% \mathrm{PEG}_{750}$-Cer-C8, $91 \mathrm{~nm}(\mathrm{pdi}=0.4)$ for BLPs with $2.7 \% \mathrm{PEG}_{750}-\mathrm{Cer}-\mathrm{C} 8$, and $122 \mathrm{~nm}$ (pdi $=0.4$ ) for BLPs with $1.4 \%$ PEG $_{2000} \mathrm{DSGS}$.

Particle analysis by TEM (Figure 1B-D), showed that BLPs formulated with $\mathrm{PEG}_{750}$-ceramides exhibited a spherical shape and revealed the presence of 2 to 5 encapsulated QDs per lipid particle (Figure 1B,C). The diameter of the BLPs varied from 100 to $130 \mathrm{~nm}$, depending on the number of loaded QDs. Empty BLPs, present as a minor fraction, and those containing only one QD were generally smaller than $100 \mathrm{~nm}$. The observed sizes for individual particles were in agreement with the mean hydrodynamic diameters obtained by DLS. Interestingly, the BLPs formulated with $1.4 \% \mathrm{PEG}_{2000}$-DSGS appeared amorphous in shape with a more variable number of loaded QDs (Figure 1D) and broader size distribution as compared to BLPs formulated with PEG-Cer-C8.

Cellular Uptake of EGFR Targeted and Two-Color QDs Labeled BLPs. Complexes of streptavidin-conjugated quantum dots (QDs) with biotin-EGF are biochemically competent ligands for erbB1, the EGF receptor (19). The first concern to be addressed was whether the internalization BLPs tagged with preformed EGF-QDs complexes could be impaired, as the large size of the lipid carrier $(\sim 100 \mathrm{~nm})$ may interfere with the dimerization of the EGFR necessary for the subsequent activation and internalization by endocytosis.

BLPs loaded with $\mathrm{QD}_{655}$ were conjugated to preformed complexes of biotin-EGF and StAv-QD 525 (EGF-QD 252 ). On the basis of previous results in which BLPs were labeled with two

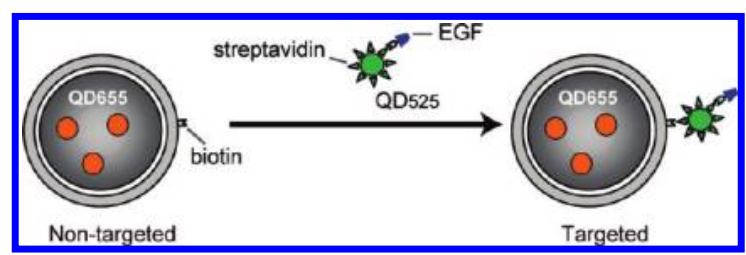

Figure 2. Scheme depicting the targeting and dual color labeling strategy of BLPs with encapsulated $\mathrm{QD}_{655}$. Preformed complexes of EGF-QD $_{525}$ (molar ratio 4:1) are conjugated to biotin molecules on the BLP surface. Specific uptake is evidenced as colocalized dots by confocal fluorescence microscopy. Red fluorescence indicate unspecific binding of nontargeted BLP-QD 655 . The binding of free unconjugated EGF-QD ${ }_{525}$, if present, is detected as green dots.

colors of streptavidin-QDs on the surface, we assumed that at least two biotins were available per lipid particle (34). In Figure 2 , the scheme depicts nontargeted BLPs loaded with $\mathrm{QD}_{655}$ (red) and the targeted and two-color labeled BLPs (red-green). With this approach, colocalized red-green dots were expected to indicate specific uptake, whereas the sole detection of red fluorescence would reveal nonspecific binding of nontargeted BLPs-QD 655 . Additionally, the two-color QD labeling strategy allowed the independent monitoring of the fate of the loaded $\mathrm{QD}_{655}$ and also circumvented the problem of purification of targeted BLPs from free EGF-QD 525 complexes. Any residual free preformed complexes of EGF-QD 525 competing for the EGFR with the targeted EGF-QD $525-$ BLPs-QD $_{655}$ upon incubation with cells could be identified easily as isolated green dots.

We employed confocal fluorescence microscopy in live cells to distinguish between specific and nonspecific uptake of BLPs by simultaneous tracking the two colors of QDs using a single excitation wavelength (Figure 3 ). The interaction of targeted and nontargeted BLPs with A431 cells overexpressing the EGFR was evaluated for BLPs formulated with $1.4 \%$ and $2.7 \% \mathrm{PEG}_{750^{-}}$ Cer-C8 and with $1.4 \%$ PEG $_{2000}$-DSGS upon incubation for $1 \mathrm{~h}$ at $37{ }^{\circ} \mathrm{C}$. Live cells were monitored for up to $2 \mathrm{~h}$ without detectable photobleaching. As shown in Figure 3, both QDs extensively colocalized (yellow spots in overlay) after $1 \mathrm{~h}$ at $37^{\circ} \mathrm{C}$ and during the $2 \mathrm{~h}$ live cell imaging. The BLPs appeared in a characteristic dot-pattern distribution, attributed to the clustering of the lipid particles in endosomal compartments, indicating that the targeted EGF-QD $525-\mathrm{BLP}_{-} \mathrm{QD}_{655}$ particles were internalized (Figure 3, panels A, B, C, left side). Negligible internalization as evidenced by a faint red fluorescence was detected for nontargeted BLP-QD 655 for all lipid formulations examined (Figure 3, panels A, B, C, right side). Similar experiments with nontargeted $\mathrm{QD}_{525}$ showed negligible binding (data not shown).

Intracellular Distribution of Internalized BLPs and Fate of $\mathbf{Q D}_{\mathbf{6 5 5}}$. The dot pattern distribution was analyzed by z-scan with serial confocal slices in representative cells. Targeted BLPs (2.7\% $\mathrm{PEG}_{750}$-Cer-C8) were internalized by A431 cells after a $10 \mathrm{~min}$ pulse followed by $1 \mathrm{~h}$ incubation (chase) at $37^{\circ} \mathrm{C}$ (Figure $4 \mathrm{~A}-\mathrm{C}$ ). Panel A shows that most of the internalized EGF$\mathrm{QD}_{525}-\mathrm{BLP}-\mathrm{QD}_{655}$ complexes accumulated in the perinuclear region of the cell, although a large fraction was still visible in small structures underneath the cell membrane (Figure 4B). Close observation of single vesicles (Figure 4D) showed a variable QD fluorescence intensity in the colocalized spots evidenced by the heterogeneous yellow-orange pseudocolor. This finding could be explained by the distribution in the number of encapsulated QDs (1-5) in BLP shown by TEM, and presumably also due to the presence of a variable number of EGF-QD ${ }_{525}$ bound to the multivalent BLPs. In addition, and possibly most important, there could have been fusion of vesicles occurring during endosomal sorting.

In Figure 4E, the fluorescence intensity profile along the line traced in panel D shows the relative contributions of green and 


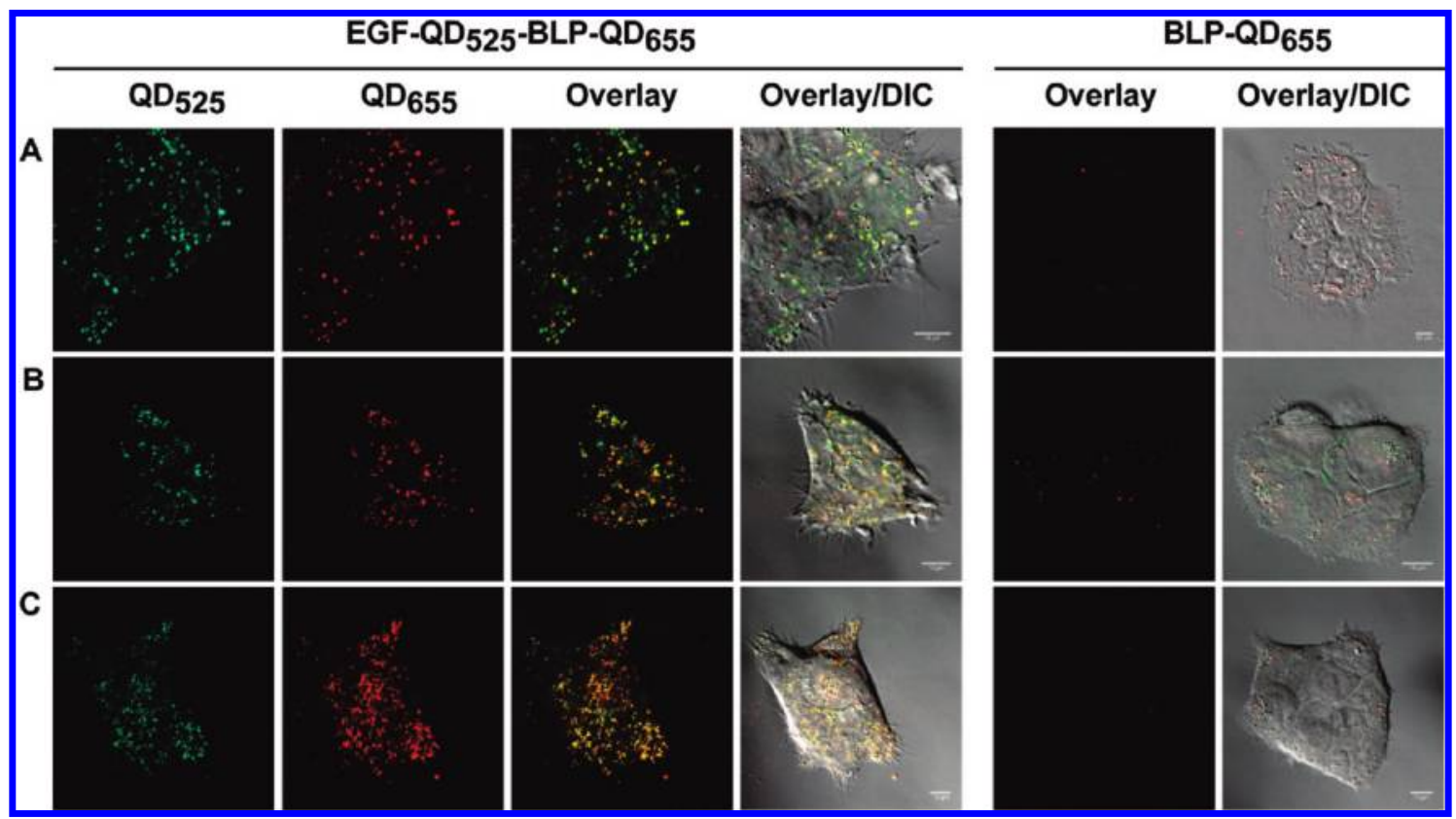

Figure 3. Cellular uptake of targeted EGF-QD $525-B_{L P}-\mathrm{QD}_{655}$ and nontargeted BLP-QD $655 . \mathrm{A} 431$ cells incubated with $\mathrm{BLPs}$ for $1 \mathrm{~h}$ at $37{ }^{\circ} \mathrm{C}$ and monitored by confocal fluorescence microscopy for up to $2 \mathrm{~h}$. Colocalization of $\mathrm{QD}_{525}$ and $\mathrm{QD}_{655}$ showed targeted BLPs distributed in endosomal compartments (left 12 panels). Nontargeted BLPs shown in right 6 panels. (A) BLPs containing $1.4 \%$ PEG $750^{-}$Cer-C8. (B) BLPs containing 2.7\% $\mathrm{PEG}_{750}$-Cer-C8. (C) BLPs containing 1.4\% PEG $_{2000}$-DSGS. Scale bar: $10 \mu \mathrm{m}$.

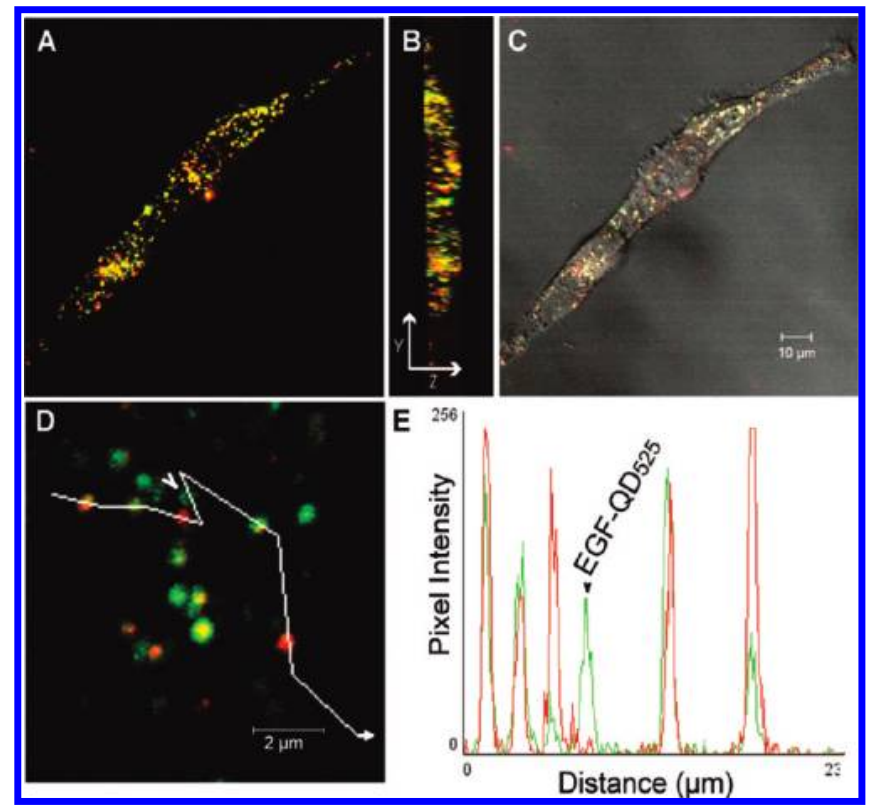

Figure 4. Intracellular fate of EGFR targeted and QDs labeled BLPs. (A) 3-D reconstruction of two A431 cells with internalized BLPs after 10 min pulse incubation and $1 \mathrm{~h}$ chase at $37^{\circ} \mathrm{C}$. (B) $y-z$ plane showing colocalized QDs inside cells and underneath the cell membrane. (C) Overlay of image A with DIC image. (D) Line-profile (in white) across six endosomal vesicles. (E) Fluorescence intensity along the line-profile in D revealing different intensity levels for colocalized QDs. Internalized two-color QDs labeled and targeted BLPs containing 2.7\% $\mathrm{PEG}_{750^{-}}$ Cer-C8. (yellow-orange pseudo color) were distinguished from independently internalized green EGF-QD 525 complexes (arrowheads in panels $\mathrm{D}$ and $\mathrm{E}$ ).

red QDs to the colocalized loci. The presence of single green dots indicates the binding of free preformed complexes of EGF$\mathrm{QD}_{525}$. These complexes were expected to be present, as they were not eliminated after conjugation with BLPs.

The BLPs formulated with a $\mathrm{pH}$-sensitive PEGylated lipid or with exchangeable $\mathrm{PEG}_{750}$-CerC8 lipids were expected to fuse more readily with the endosomal membranes, thereby

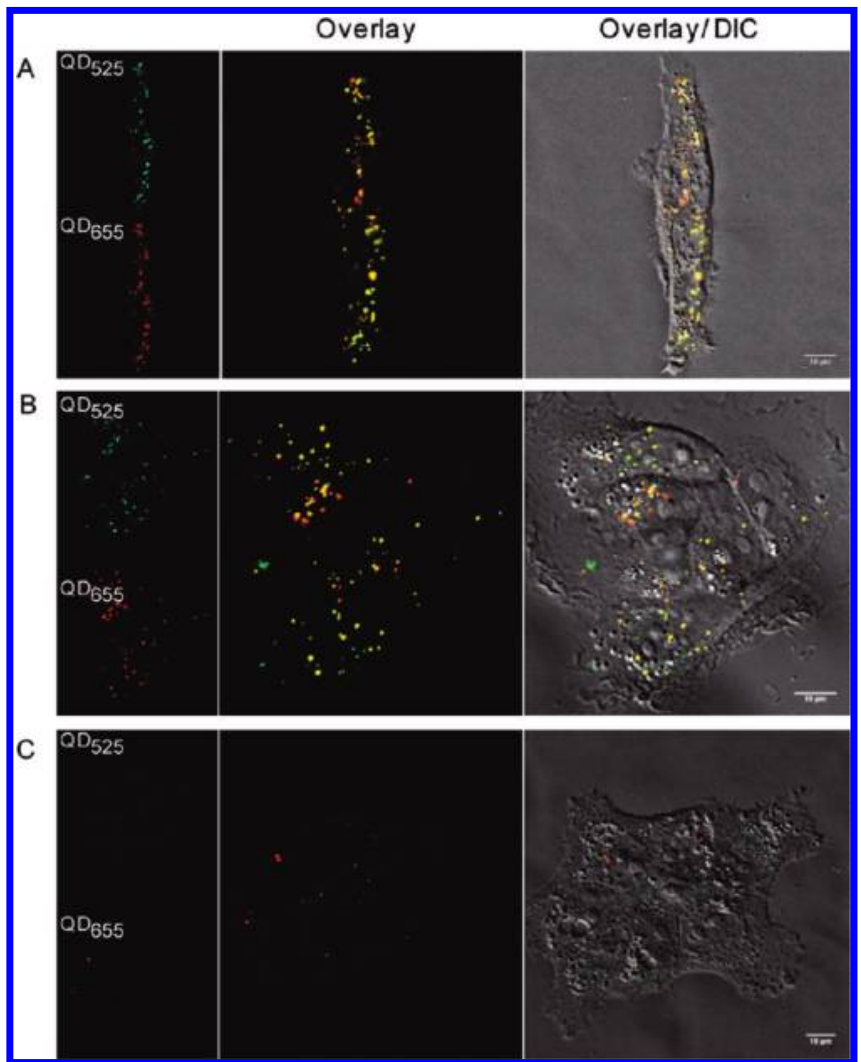

Figure 5. Fate of encapsulated $\mathrm{QD}_{655}$ delivered by EGF-QD $\mathrm{D}_{52}-\mathrm{BLP}-$ $\mathrm{QD}_{655}$. After $10 \mathrm{~min}$ incubation at $37^{\circ} \mathrm{C}$ with $\mathrm{A} 431$ cells, unbound EGF-QD $525-$ BLP-QD $_{655}$ were removed and cells imaged by confocal microspcopy after $4 \mathrm{~h}(\mathrm{~A})$ and $12 \mathrm{~h}(\mathrm{~B})$, nontargeted BLP-QD $655(\mathrm{C})$. BLP containing $2.7 \%$ PEG $_{750}$-Cer-C8. Scale bar $10 \mu \mathrm{m}$.

releasing the QDs into the cytoplasm. Consequently, the initial colocalization observed for the dual-labeled EGF-QD $525-B L P-$ $\mathrm{QD}_{655}$ was expected to decrease in time. However, as shown in Figure 5 , after 4 and $12 \mathrm{~h}$ chase of the BLPs at $37{ }^{\circ} \mathrm{C}$ dissociation of the two QD signals was not apparent. The dot 

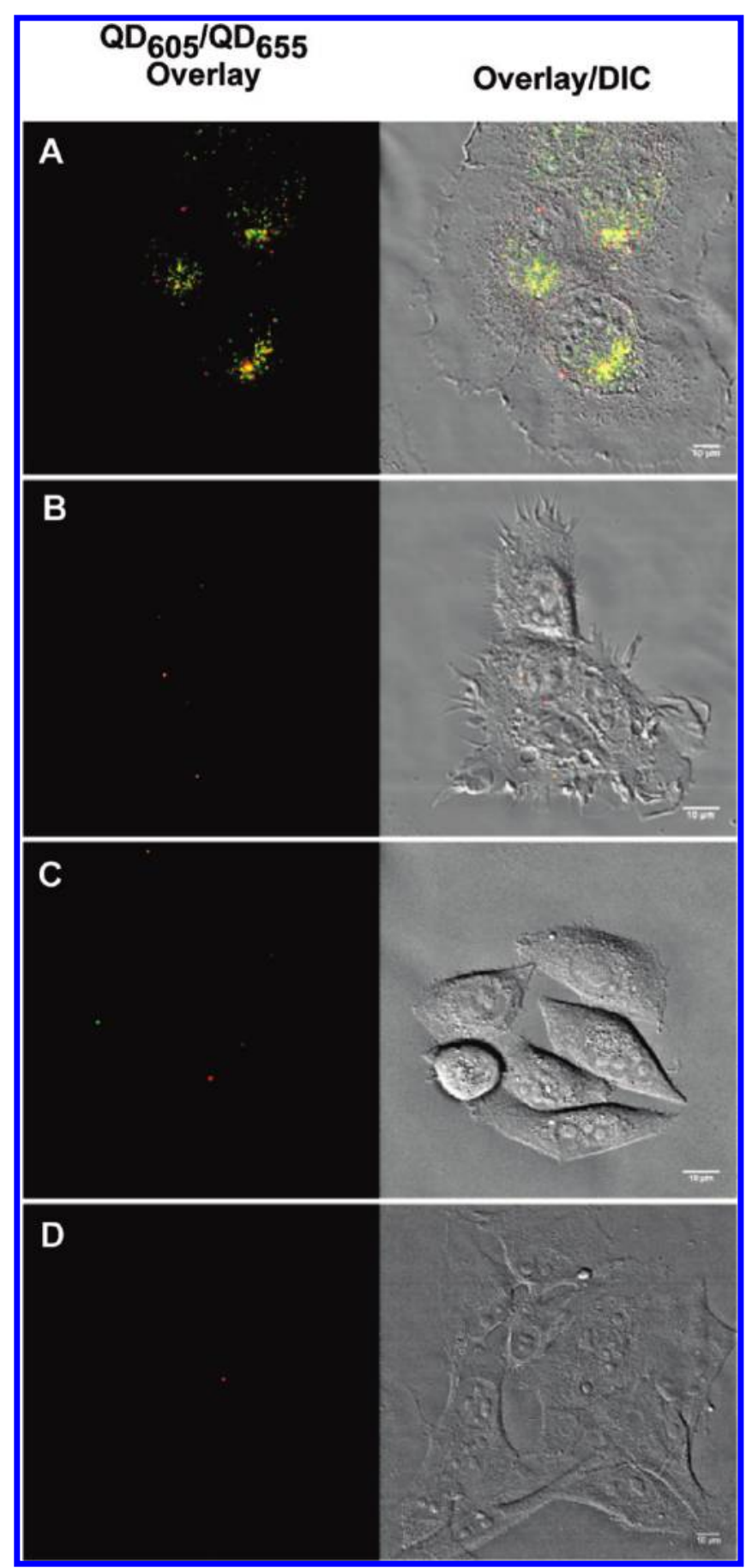

Figure 6. Binding specificity of EGFR targeted BLPs. A431 cells incubated with targeted BLP during a 10 min pulse at $37^{\circ} \mathrm{C}$ and chased after 60 min (A) with EGF-QD $605-B_{1}-Q_{-15}$, (B) coincubated with EGF-QD $605-\mathrm{BLP}-\mathrm{QD}_{655}$ and a 16-fold molar excess of free competing ligand EGF. (C) CHO cells devoid of EGFR incubated with EGF$\mathrm{QD}_{605}-\mathrm{BLP}_{-} \mathrm{DD}_{655}$. (D) WM983A cells devoid of EGFR incubated with EGF-QD 605 -BLP-QD 655 . BLP: 2.7\% PEG $_{750}$-Cer-C8. Scale bar $10 \mu \mathrm{m}$.

pattern persisted, suggesting that QDs remain trapped in BLPs in endolysosomal compartments.

Specific Binding of EGFR Targeted and QD Labeled BLPs. The specificity of targeting was examined in a competitive binding assay (Figure 6A,B) carried out by incubating the targeted and labeled BLPs with A431 cells in the presence of a 16-fold molar excess of free ligand EGF (panel B). Under these conditions, the absence of colocalized QDs indicated that the binding of the targeted BLPs was substantially diminished as compared to the positive controls performed without compet- ing ligand (panel A). In addition, targeted BLPs were incubated with EGFR-negative cells. Neither CHO cells nor WM983A cells internalized the EGFR targeted BLPs as evidenced by the absence of colocalized dots in Figure 6C,D. Taken together, these results indicate that the enhanced uptake of targeted BLP was due to the specific interaction of the lipid-bound QD-EGF with EGFR and not by macropinocytosis induced by non-lipidbound QD-EGF.

\section{DISCUSSION}

An emerging tool in targeted delivery is the multimodal carrier, in which a therapeutic molecule, an imaging agent, and a targeting entity are assembled in a single nanoparticle for simultaneous imaging and therapy $(25,27,30,35)$. In the present work, we developed a QD-labeled and EGFR targeted delivery system mediated by biotinylated lipid particles (BLPs). The originality of our approach lies in the novel encapsulation of QDs in BLPs by a detergent dialysis technique, where the inclusion of as little as 1.4 mol \% PEG-lipids allowed the selfassembly of stable BLPs. The sucrose density gradient proved very useful for the separation of QD-loaded BLPs from free QDs (Figure 1A). TEM analysis of the purified fractions showed an average of 2-5 encapsulated QDs in $~ 100$-nm-sized lipid vesicles (Figure 1B,C,D). This size was in agreement with the mean diameters obtained by DLS for all lipid formulations.

A dual-color labeling strategy with loaded and surface-bound QDs was introduced in order to follow the intracellular localization of the cargo and carrier by colocalization analysis using confocal microscopy. BLP-QD 655 were surface modified with preformed complexes of EGF-QD 525 and delivered to A431 cells overexpressing the EGFR. Addition of the biotin-EGF ligand as a preformed complex with StAvQDs instead of direct binding to BLPs was adopted to improve ligand-receptor binding, taking advantage of the multiple streptavidins coating the QDs (6-8 streptavidins according to the manufacturer). The application of these preformed complexes extends the use to any biotinylated ligand. The two-color labeled and targeted EGF$\mathrm{QD}_{525}$-BLP-QD 655 were internalized by the A431 cells, whereas nontargeted BLP-QD 655 showed negligible binding, indicating that uptake of the particles was mediated by the EGFR activation and internalization (Figure 3). The specificity of targeted BLPs for the EGF receptor was supported by a competitive binding assay performed in the presence of 16-fold molar excess of free ligand EGF (Figure 6A,B). Under these conditions, binding to A431 cells was completely abolished. In line with these observations, CHO cells and WM983A melanoma cells that do not express the EGFR showed no binding of the targeted particles (Figure 6C,D).

Upon internalization of EGF-QD $525-B_{2}-Q_{655}$, the variations in the relative fluoresecence intensities found for colocalized QDs (Figure 4) were interpreted as the result of an uneven distribution in the number of encapsulated $\mathrm{QD}_{655}$ and EGF$\mathrm{QD}_{525}$ complexes conjugated to individual BLP. Additionally, QDs fluorescence intensity variation could be due to endosomal fusion bringing several BLPs together.

We were also interested in the cytoplasmic delivery of the encapsulated $\mathrm{QD}_{655}$. Therefore, BLPs were formulated with 1.4 and $2.7 \mathrm{~mol} \%$ PEG conjugated to fusogenic or to $\mathrm{pH}$-sensitive lipids in order to destabilize BLPs bilayers and facilitate fusion with endosomes $(8,10,11)$. On the basis of the dual color labeling strategy, the cytoplasmic release of $\mathrm{QD}_{655}$ was expected to be evidenced by delocalization of initially colocalized red-green dots However, the dot pattern of colocalized QDs persisted for up to $12 \mathrm{~h}$, regardless of the formulation tested and PEG content. The possible explanations would be that either PEG coating was not completely removed or that QDs aggregated inside the acidic environment of endosomes due to 
protonation of carboxyl groups and charge neutralization on QD surfaces. Several approaches have been devised for delivering QDs intracellularly. They include conjugation of QDs to cell penetrating peptides $(36,37)$ and encapsulation in targeted micelles (25) or polymers (38). However, QDs remain predominantly trapped in endosomal compartments and only direct microinjection (23) into cell cytoplasm and more recently osmotic lysis of pinosomes $(21,22)$ appear as the methods of choice for QD delivery with homogeneous distribution.

The two-color QDs labeling strategy allowed a clear distinction to be made between the targeted and $\mathrm{QD}_{655}$-loaded BLPs (yellow-orange pseudocolors) and the independently internalized green EGF-QD ${ }_{525}$ complexes (Figure 4D,E), avoiding the tedious purification of targeted BLPs from free EGF-QDs complexes. During the continuous live cell imaging for up to $2 \mathrm{~h}$, photobleaching was undetectable, highlighting the photostability of QDs as probes for long-term imaging of dynamic processes.

In the developed lipid carrier, the multivalency of BLPs and StAvQDs serves to increase the number of EGF ligands on the BLP surface. However, variations in size and, as a consequence, the number of biotins in/on individual BLPs influence the controlled and stoichiometric addition of preformed EGF-QD complexes to BLPs. Thus, further refinements of the system are desirable. Nonethless, it is evident that the addition of quantum dots into liposomal carriers, fulfilling a dual role as cargo and surface tag, provides a flexible platform for cell targeting and monitoring receptor-mediated uptake employing any biotinylated ligand attached to StAv-QDs. The expectation is that such targeted lipid-based delivery systems will constitute a new means for the effective cytoplasmic delivery of therapeutic macromolecules.

\section{ACKNOWLEDGMENT}

This work was financially supported by the Max Planck Society, the DFG Research Center for Molecular Physiology of the Brain (CMPB) -Excellence Cluster EXC 171 - FZT103 "Microscopy at the Nanometer Range", and the EU sixth framework (FP6) project FLUOROMAG, LHSB-CT-2006037465. The authors thank Dr. Dietmar Riedel for conducting the TEM measurements.

Note Added after ASAP Publication: The version of this paper that was published on the Web on July 22, 2010, did not contain all of the author's corrections. The corrected version was reposted on July 27, 2010.

Supporting Information Available: Fluorometric assay for biotin quantitation and characterization of individual BLPs preparations. This material is available free of charge via the Internet at http://pubs.acs.org.

\section{LITERATURE CITED}

(1) Harris, J. M, and Chess, R. B. (2003) Effect of pegylation on pharmaceuticals. Nat. Rev. Drug Discovery 2, 214-21.

(2) Woodle, M. C., and Lasic, D. D. (1992) Sterically stabilized liposomes. Biochim. Biophys. Acta 1113, 171-99.

(3) Holland, J. W., Hui, C., Cullis, P. R., and Madden, T. D. (1996) Poly(ethylene glycol)-lipid conjugates regulate the calciuminduced fusion of liposomes composed of phosphatidylethanolamine and phosphatidylserine. Biochemistry 35, 2618-24.

(4) Kirpotin, D., Hong, K., Mullah, N., Papahadjopoulos, D., and Zalipsky, S. (1996) Liposomes with detachable polymer coating: destabilization and fusion of dioleoylphosphatidylethanolamine vesicles triggered by cleavage of surface-grafted poly(ethylene glycol). FEBS Lett. 388, 115-8.
(5) Choi, J. S., MacKay, J. A., and Szoka, F. C., Jr. (2003) LowpH-sensitive PEG-stabilized plasmid-lipid nanoparticles: preparation and characterization. Bioconiugate Chem. 14, 420-9.

(6) Guo, X., MacKay, J. A., and Szoka, F. C., Jr. (2003) Mechanism of $\mathrm{pH}$-triggered collapse of phosphatidylethanolamine liposomes stabilized by an ortho ester polyethyleneglycol lipid. Biophvs. J. 84, 1784-95.

(7) Guo, X., and Szoka, F. C., Jr. (2001) Steric stabilization of fusogenic liposomes by a low-pH sensitive PEG-diortho esterlipid conjugate. Bioconiugate Chem. 12, 291-300.

(8) Shi, F., Wasungu, L., Nomden, A., Stuart, M. C., Polushkin, E., Engberts, J. B., and Hoekstra, D. (2002) Interference of poly(ethylene glycol)-lipid analogues with cationic-lipid-mediated delivery of oligonucleotides; role of lipid exchangeability and non-lamellar transitions. Biochem. J. 366, 333-41.

(9) Wheeler, J. J., Palmer, L., Ossanlou, M., MacLachlan, I., Graham, R. W., Zhang, Y. P., Hope, M. J., Scherrer, P., and Cullis, P. R. (1999) Stabilized plasmid-lipid particles: construction and characterization. Gene Ther. 6, 271-81.

(10) Mok, K. W., Lam, A. M., and Cullis, P. R. (1999) Stabilized plasmid-lipid particles: factors influencing plasmid entrapment and transfection properties. Biochim. Biophys. Acta 1419, 13750 .

(11) Li, W., Huang, Z., MacKay, J. A., Grube, S., and Szoka, F. C., Jr. (2005) Low-pH-sensitive poly(ethylene glycol) (PEG)stabilized plasmid nanolipoparticles: effects of PEG chain length, lipid composition and assembly conditions on gene delivery. J. Gene Med. 7, 67-79.

(12) Alivisatos, A. P., Gu, W., and Larabell, C. (2005) Quantum dots as cellular probes. Annu. Rev. Biomed. Eng. 7, 55-76.

(13) Michalet, X., Pinaud, F. F., Bentolila, L. A., Tsay, J. M., Doose, S., Li, J. J., Sundaresan, G., Wu, A. M., Gambhir, S. S., and Weiss, S. (2005) Quantum dots for live cells, in vivo imaging, and diagnostics. Science 307, 538-44.

(14) Smith, A. M., Gao, X., and Nie, S. (2004) Quantum dot nanocrystals for in vivo molecular and cellular imaging. Photochem. Photobiol. 80, 377-85.

(15) Kirchner, C., Liedl, T., Kudera, S., Pellegrino, T., Muñoz, J. A., Gaub, H. E., Stolzle, S., Fertig, N., and Parak, W. J. (2005) Cytotoxicity of colloidal $\mathrm{CdSe}$ and $\mathrm{CdSe} / \mathrm{ZnS}$ nanoparticles. Nano Lett. 331-338.

(16) Al-Jamal, W. T., and Kostarelos, K. (2007) Liposomenanoparticle hybrids for multimodal diagnostic and therapeutic applications. Nanomedicine 2, 85-98.

(17) Yarden, Y. (2001) The EGFR family and its ligands in human cancer. signalling mechanisms and therapeutic opportunities. Eur. J. Cancer 37 Suppl 4, S3-8.

(18) Lidke, D. S., Nagy, P., Heintzmann, R., Arndt-Jovin, D. J., Post, J. N., Grecco, H. E., Jares-Erijman, E. A., and Jovin, T. M. (2004) Quantum dot ligands provide new insights into erbB/ HER receptor-mediated signal transduction. Nat. Biotechnol. 22, 198-203.

(19) Lidke, D. S., Nagy, P., Jovin, T. M., and Arndt-Jovin, D. J. (2007) Biotin-ligand complexes with streptavidin quantum dots for in vivo cell labeling of membrane receptors. Methods Mol. Biol. 374, 69-79.

(20) Sperling, R., Pellegrino, T., Li, J. K., Chang, W. H., and Parak, W. J. (2006) Electrophoretic separation of nanoparticles with a discrete number of functional groups. Adv. Funct. Mater. 26, 943-948.

(21) Courty, S., Bouzigues, C., Luccardini, C., Ehrensperger, M. V., Bonneau, S., and Dahan, M. (2006) Tracking individual proteins in living cells using single quantum dot imaging. Methods Enzymol. 414, 211-28.

(22) Nelson, R. S., Ali, M. Y., Trybus, K. M., and Warshaw, D. M. (2009) Random walk of processive, quantum dot-labeled myosin va molecules within the actin cortex of COS-7 cells. Biophvs. J. 97, 509-518.

(23) Derfus, A. M., Chan, W. C. W., and Bhatia, S. N. (2004) Intracellular delivery of quantum dots for live cell labeling and organelle tracking. Adv. Mater. 16, 961-966. 
(24) Bothun, G. D., Rabideau, A. E., and Stoner, M. A. (2009) Hepatoma cell uptake of cationic multifluorescent quantum dot liposomes. J. Phys. Chem. B 113, 7725-8.

(25) Rubinstein, I., Soos, I., and Onyuksel, H. (2008) Intracellular delivery of VIP-grafted sterically stabilized phospholipid mixed nanomicelles in human breast cancer cells. Chem. Biol. Interact. $171,190-4$.

(26) Al-Jamal, W. T., Al-Jamal, K. T., Tian, B., Lacerda, L., Bomans, P. H., Frederik, P. M., and Kostarelos, K. (2008) Lipidquantum dot bilayer vesicles enhance tumor cell uptake and retention in vitro and in vivo. ACS Nano 2, 408-18.

(27) Schroeder, J. E., Shweky, I., Shmeeda, H., Banin, U., and Gabizon, A. (2007) Folate-mediated tumor cell uptake of quantum dots entrapped in lipid nanoparticles. $\underline{\text { J. Controlled }}$ Release 124, 28-34.

(28) Dubertret, B., Skourides, P., Norris, D. J., Noireaux, V., Brivanlou, A. H., and Libchaber, A. (2002) In vivo imaging of quantum dots encapsulated in phospholipid micelles. Science 298 , 1759-62.

(29) Maysinger, D., Lovric, J., Eisenberg, A., and Savic, R. (2007) Fate of micelles and quantum dots in cells. Eur. J. Pharm. Biopharm. 65, 270-81.

(30) Weng, K. C., Noble, C. O., Papahadjopoulos-Sternberg, B., Chen, F. F., Drummond, D. C., Kirpotin, D. B., Wang, D., Hom, Y. K., Hann, B., and Park, J. W. (2008) Targeted tumor cell internalization and imaging of multifunctional quantum dot-conjugated immunoliposomes in vitro and in vivo. Nano Lett. 8, 2851-7.
(31) Sabharwal, N., Holland, E. C., and Vazquez, M. (2009) Live cell labeling of glial progenitor cells using targeted quantum dots. Ann. Biomed. Eng. 37, 1967-1973.

(32) Roberti, M. J., Morgan, M., Menendez, G., Pietrasanta, L. I., Jovin, T. M., and Jares-Erijman, E. A. (2009) Quantum dots as ultrasensitive nanoactuators and sensors of amyloid aggregation in live cells. J. Am. Chem. Soc. 131, 8102-7.

(33) Batchelor, R. H., Sarkez, A., Cox, W. G., and Johnson, I. (2007) Fluorometric assay for quantitation of biotin covalently attached to proteins and nucleic acids. Biotechniques 43, $503-7$.

(34) Sigot, V., and Jovin, T. M. (2008) Quantum dots: torch bearers into cells. Imaging Microsc. 10, 40-43.

(35) Qi, L., and Gao, X. (2008) Emerging application of quantum dots for drug delivery and therapy. Expert Opin. Drug Deliverv $5,263-7$.

(36) Lagerholm, B. C. (2007) Peptide-mediated intracellular delivery of quantum dots. Methods Mol. Biol. 374, 105-12.

(37) Medintz, I. L., Pons, T., Delehanty, J., Susumu, K., Brunel, F., Dawson, P., and Mattoussi, H. (2008) Intracellular delivery of quantum dot-protein cargos mediated by cell penetrating peptides. Bioconiugate Chem. 9, 1785-1795.

(38) Duan, H., and Nie, S. (2007) Cell-penetrating quantum dots based on multivalent and endosome-disrupting surface coatings. J. Am. Chem. Soc. 129, 3333-8.

BC100054C 